\title{
A BRIEF PROOF OF CAUCHY'S INTEGRAL THEOREM
}

\author{
JOHN D. DIXON ${ }^{1}$
}

\begin{abstract}
A short proof of Cauchy's theorem for circuits homologous to 0 is presented. The proof uses elementary local properties of analytic functions but no additional geometric or topological arguments.
\end{abstract}

The object of this note is to present a very short and transparent proof of Cauchy's theorem for circuits homologous to 0 . The proof is based on simple 'local' properties of analytic functions that can be derived from Cauchy's theorem for analytic functions on a disc, and it may be compared with the treatment in Ahlfors [1, pp. 137-145]. It is apparent from this proof that this version of Cauchy's theorem is not only much more natural than the homotopic version which appears in several recent textbooks; it is also much easier to prove (contra Dieudonné [2, p. 192]). It is reasonable to argue that the concept of homotopy in connection with Cauchy's theorem is as extraneous as the notion of Jordan curve.

We recall that if $\gamma$ is a circuit (= "continuous, piecewise smooth, closed curve"), and $w \in C$ does not lie on $\gamma$, then the index of $w$ with respect to $\gamma$ is $\operatorname{Ind}(\gamma, w)=(2 \pi i)^{-1} \int_{\gamma}(z-w)^{-1} d z$. It is easily proved that $E=\{w \in C \mid \operatorname{Ind}(\gamma, w)=0\}$ contains a neighbourhood of $\infty$ and is open (see [1, p. 116]). In the following proof we give full references to the 'local' properties used in order to emphasize the elementary nature of the proof.

Cauchy's theorem. Let $D$ be an open subset of $\mathrm{C}$ and let $\gamma$ be $a$ circuit in $D$. Suppose that $\gamma$ is homologous to 0 in $D$, i.e. each $w \in D$ lies in the set $E$ defined above. Then, for each $f$ analytic on $D$ :

(i) $\int_{\gamma} f(z) d z=0$;

(ii) $\operatorname{Ind}(\gamma, w) f(w)=(2 \pi i)^{-1} \int_{\gamma}(z-w)^{-1} f(z) d z$ for all $w \in D$ not lying on $\gamma$.

Proof. Consider $g: D \times D \rightarrow C$ defined by $g(w, z)=(f(z)-f(w)) /(z-w)$ for $z \neq w$ and $g(w, w)=f^{\prime}(w)$. Then $g$ is continuous, and for each fixed $z, w \mapsto g(w, z)$ is analytic $[1$, p. 124]. Define $h: C \rightarrow C$ by $h(w)$

Received by the editors January 18, 1971.

AMS 1970 subject classifications. Primary 30X01, 30A86.

Key words and phrases. Cauchy's integral theorem, Cauchy's integral formula, residue theorem.

1 This research was supported in part by the National Research Council of Canada (Grant No. A7171).

Copyright (c) 1971, American Mathematical Society 
$=\int_{\gamma} g(w, z) d z$ on $D$ and $h(w)=\int_{\gamma}(z-w)^{-1} f(z) d z$ on $E$. Note that $\boldsymbol{C}=D \cup E$ by hypothesis, and that these two expressions for $h(w)$ are equal on $D \cap E$ because $\operatorname{Ind}(\gamma, w)=0$ there.

Now $h$ is differentiable on both $D$ and $E$ ([1, p. 123] or [3, p. 137]), and so $h$ is an entire function. Since the image of $\gamma$ is bounded, and $E$ contains a neighbourhood of $\infty, h(w) \rightarrow 0$ as $w \rightarrow \infty$. This implies firstly that $h$ is constant (Liouville's theorem), and secondly that $h$ is 0 . Thus $\int_{\gamma} g(w, z) d z=0$ for all $w \in D$ not lying on $\gamma$; and (ii) follows. Finally, let $u$ be some fixed point of $D$ not lying on $\gamma$. Then applying (ii) to the function $z \mapsto f(z)(z-u)$ in place of $f$, and evaluating at $w=u$, we obtain (i).

REMARK. The proof goes through word for word when $\gamma$ is a cycle (see [1, p. 138]) rather than a circuit. Then, as in Ahlfors' treatment, the general form of the residue theorem follows immediately.

\section{REFERENCES}

1. L. V. Ahlfors, Complex analysis: An introduction to the theory of analytic functions of one complex variable, 2nd ed., McGraw-Hill, New York, 1966. MR 32 \#5844.

2. J. Dieudonné, Foundations of modern analysis, Pure and Appl. Math., vol. 10, Academic Press, New York, 1960. MR 22 \#11074.

3. R. Nevanlinna and V. Paatero, Einführung in die Funktionentheorie, Lehrbücher und Monographien aus dem Gebiete der exakten Wissenschaften, Mathematische Reihe, Band 30, Birkhäuser Verlag, Basel, 1965; English transl., AddisonWesley, Reading, Mass., 1969. MR 34 \#1491; MR 39 \#415.

Carleton University, Ottawa 1, Ontario, Canada 